\title{
Numerical Simulations for the Space-Time Variable Order Nonlinear Fractional Wave Equation
}

\author{
Nasser Hassan Sweilam ${ }^{1}$ and Taghreed Abdul Rahman Assiri ${ }^{2}$ \\ ${ }^{1}$ Department of Mathematics, Faculty of Science, Cairo University, Giza 12613, Egypt \\ ${ }^{2}$ Department of Mathematics, Faculty of Science, Umm Al-Qura University, Makkah 21955, Saudi Arabia \\ Correspondence should be addressed to Nasser Hassan Sweilam; n_sweilam@yahoo.com
}

Received 3 March 2013; Revised 29 April 2013; Accepted 3 May 2013

Academic Editor: Chein-Shan Liu

Copyright (c) 2013 N. H. Sweilam and T. A. R. Assiri. This is an open access article distributed under the Creative Commons Attribution License, which permits unrestricted use, distribution, and reproduction in any medium, provided the original work is properly cited.

\begin{abstract}
The explicit finite-difference method for solving variable order fractional space-time wave equation with a nonlinear source term is considered. The concept of variable order fractional derivative is considered in the sense of Caputo. The stability analysis and the truncation error of the method are discussed. To demonstrate the effectiveness of the method, some numerical test examples are presented.
\end{abstract}

\section{Introduction}

It is well known that the fractional calculus definitions are extensions of the usual calculus definitions [1-8], where the orders need not to be positive integers. On the other hand, the variable order calculus is a natural extension of the constant order (integer or fractional) calculus. In this sense, the order may function in any variable such as time and space variables or a system of other variables $[9,10]$. In general, one can say that this extension is introduced by Samko and Ross in [11], where Marchaud fractional derivative and Riemann-Liouville derivative are extended to the variable order cases the order in this case is a function in the space variable only. Many authors have introduced different definitions of variable order differential operators, each of these with a specific meaning to suit desired goals. These definitions such as RiemannLiouville, Grünwald, Caputo, Riesz [3, 12-16], and some notes as Coimbra definition $[17,18]$.

Coimbra in [17] used Laplace transform of Caputo's definition of the fractional derivative as the starting point to suggest a novel definition for the variable order differential operator. Because of its meaningful physical interpretation, Coimbra's definition is better suited for modeling physical problems. The variable order differentials are an important tool to study some systems such as the control of nonlinear viscoelasticity oscillator (for more details see [17-19] and the references cited therein), where the order changes with respect to a parameter or more parameters.

In the following, we present the basic definition for the variable order fractional derivatives which we will use in this paper.

Definition 1 (see [14]). The Caputo space variable order derivative is defined as follows:

$$
\begin{aligned}
D_{x}^{\propto(x, t)} u(x, t)= & \frac{1}{\Gamma(n-\propto(x, t))} \\
& \times \int_{0}^{x} \frac{1}{(x-\xi)^{\propto(x, t)-n+1}} \frac{\partial^{n} u(\xi, t)}{\partial \xi^{n}} d \xi,
\end{aligned}
$$

where $0<\propto(x, t)<1$.

The main aim of this work is to use the explicit finite difference method (EFDM) to study numerically the following nonlinear space-time variable order wave equation:

$$
\begin{array}{r}
\frac{\partial^{\beta(x, t)} u(x, \tau)}{\partial \tau^{\beta(x, t)}}=B(x, t) \frac{\partial^{\propto(x, t)} u(x, \tau)}{\partial x^{\propto(x, t)}}+f(u, x, t), \\
1<\propto(x, t), \beta(x, t) \leq 2,
\end{array}
$$

subject to initial conditions

$$
u(x, 0)=\varphi_{1}(x), \quad u_{t}(x, 0)=\varphi_{2}(x),
$$


and the following boundary conditions

$$
u(0, t)=\Psi_{1}(x), \quad u(a, t)=\Psi_{2}(x),
$$

where $0 \leq x<a, 0 \leq t<T, B(x, t)>0$ is a constant, $\Psi_{1}(x), \Psi_{2}(x)$ are smooth functions, and $f(u, x, t)$ is a nonlinear scour term that satisfies the Lipschitz condition, that is,

$$
\left|f\left(u_{1}, x, t\right)-f\left(u_{2}, x, t\right)\right| \leq L\left|u_{1}-u_{2}\right|,
$$

where the constant $L>0$ is called a Lipschitz constant for $f$.

\section{Discretization for EFDM}

In this section, EFDM is used to study the model problem (2), then the space-time solutions domain will be discretized. The discrete form for the pervious Caputo derivative can be written as follows:

$$
\begin{aligned}
& D_{x}^{\propto(x, t)} u(x, t) \\
&=\frac{1}{\Gamma(2-\propto(x, t))} \\
& \quad \times \int_{0}^{x} \frac{1}{(x-\xi)^{\propto(x, t)-2+1}} \frac{\partial^{2} u(\xi, t)}{\partial \xi^{2}} d \xi \\
&=\frac{1}{\Gamma(2-\propto(x, t))} \sum_{k=0}^{i-1} \int_{k h}^{(k+1) h} z^{1-\propto(x, t)} \\
& \quad \times \frac{\partial^{2} u(x-z, \tau)}{\partial z^{2}} d z, \quad z=x-\xi \\
& \approx \frac{1}{\Gamma(2-\propto(x, t))} \\
& \times \sum_{k=0}^{i-1}((u(x-(k-1) h, t)-2 u(x-k h, t) \\
& \times \int_{k h}^{(k+1) h} z^{1-\propto} d z . \\
&\left.+u(x-(k+1) h, t))\left(h^{2}\right)^{-1}\right)
\end{aligned}
$$

Then,

$$
\begin{aligned}
& D_{x}^{\propto(x, t)} u(x, t) \\
& \approx \frac{h^{2-\propto(x, t)}}{\Gamma(3-\propto(x, t))} \\
& \quad \times \sum_{k=0}^{i-1}((u(x-(k-1) h, t)-2 u(x-k h, t) \\
& \left.\quad+u(x-(k+1) h, t))\left(h^{2}\right)^{-1}\right) \\
& \quad \times\left((k+1)^{2-\propto(x, t)}-k^{2-\propto(x, t)}\right) .
\end{aligned}
$$

Now, pick two positive integers $N, M$ and define the step size of space and time by $h, \tau$, respectively, where $h=a / M$ and $\tau=T / N$. Also we introduce the following notations:

$$
\begin{gathered}
x_{i}=i h, \quad \text { for } i=1,2, \ldots, N, \\
t_{j}=j \tau, \quad \text { for } j=1, \ldots, M,
\end{gathered}
$$

$u_{i}^{j} \approx u\left(x_{i}, t_{j}\right), \quad B_{i}^{j}=B\left(x_{i}, t_{j}\right)$, and $f_{i}^{j}=f\left(u_{i}^{j}, x_{i}, t_{j}\right)$. Then,

$$
\begin{aligned}
D_{x}^{\propto(x, t)} u & (x, t) \\
= & \frac{h^{-\alpha_{i}^{j}}}{\Gamma\left(3-\alpha_{i}^{j}\right)} \sum_{k=0}^{i-1}\left(u_{i-k+1}^{j}-2 u_{i-k}^{j}+u_{i-k-1}^{j}\right) \\
& \times\left((k+1)^{2-\alpha_{i}^{j}}-k^{2-\alpha_{i}^{j}}\right) .
\end{aligned}
$$

By the same way, we have

$$
\begin{aligned}
D_{t}^{\beta(x, t)} & u(x, t) \\
= & \frac{\tau^{-\beta_{i}^{j}}}{\Gamma\left(3-\beta_{i}^{j}\right)} \sum_{k=0}^{j-1}\left(u_{i}^{j-k+1}-2 u_{i}^{j-k}+u_{i}^{j-k-1}\right) \\
& \times\left((k+1)^{2-\beta_{i}^{j}}-k^{2-\beta_{i}^{j}}\right) .
\end{aligned}
$$

For simplicity, let us define

$$
\begin{gathered}
R_{i}^{j}=\frac{B_{i}^{j} h^{-\propto_{i}^{j}}}{\Gamma\left(3-\propto_{i}^{j}\right)}, \quad Q_{i}^{j}=\frac{\Gamma\left(3-\beta_{i}^{j}\right)}{\tau^{-\beta_{i}^{j}}}, \\
G_{k}^{j}=\left((k+1)^{2-\propto_{i}^{j}}-k^{2-\propto_{i}^{j}}\right), \\
H_{i}^{k}=\left((k+1)^{2-\beta_{i}^{j}}-k^{2-\beta_{i}^{j}}\right) .
\end{gathered}
$$

Then, we can rewrite (2) in the following form:

$$
\begin{aligned}
& \sum_{k=0}^{j-1}\left(u_{i}^{j-k+1}-2 u_{i}^{j-k}+u_{i}^{j-k-1}\right) H_{i}^{k} \\
& \quad \approx Q_{i}^{j} R_{i}^{j} \sum_{k=0}^{i-1}\left(u_{i-k+1}^{j}-2 u_{i-k}^{j}+u_{i-k-1}^{j}\right) G_{k}^{j}+Q_{i}^{j} f_{i}^{j},
\end{aligned}
$$

that is,

$$
\begin{aligned}
u_{i}^{j+1}= & 2 u_{i}^{j}-u_{i}^{j-1} \\
& -\sum_{k=1}^{j-1}\left(u_{i}^{j-k+1}-2 u_{i}^{j-k}+u_{i}^{j-k-1}\right) H_{i}^{k} \\
& +Q_{i}^{j} R_{i}^{j} \sum_{k=0}^{i-1}\left(u_{i-k+1}^{j}-2 u_{i-k}^{j}+u_{i-k-1}^{j}\right) G_{k}^{j}+Q_{i}^{j} f_{i}^{j}, \\
u_{i}^{j+1}= & \left(2-H_{i}^{1}\right) u_{i}^{j} \\
& -\sum_{k=2}^{M-2}\left(H_{i}^{k-2}-2 H_{i}^{k-1}+H_{i}^{k}\right) u_{i}^{j-k+1} \\
& -\left(H_{i}^{j-2}-2 H_{i}^{j-1}\right) u_{i}^{1}-H_{i}^{j-1} u_{i}^{0} \\
& +Q_{i}^{j} R_{i}^{j} \sum_{k=0}^{i-1}\left(u_{i-k+1}^{j}-2 u_{i-k}^{j}+u_{i-k-1}^{j}\right) G_{k}^{j}+Q_{i}^{j} f_{i}^{j} .
\end{aligned}
$$


The previous equation can be expressed in the following matrix form:

$$
U_{i}^{0}=\emptyset_{1}, \quad U_{i}^{1}=U_{i}^{0}+\tau \emptyset_{2},
$$

and for $j \geq 2$

$$
\begin{aligned}
U_{i}^{j+1}= & A^{j} U_{i}^{j} \\
& -\sum_{k=2}^{M-2}\left(H_{i}^{k-2}-2 H_{i}^{k-1}+H_{i}^{k}\right) U_{i}^{j-k+1} \\
& -\left(H_{i}^{j-2}-2 H_{i}^{j-1}\right) U_{i}^{1}-H_{i}^{j-1} U_{i}^{0}+F^{j},
\end{aligned}
$$

where $F^{j}=\left(Q_{i}^{j} f\left(u_{m-1}^{j}, x_{m-1}, t_{j}\right), \ldots, Q_{i}^{j} f\left(u_{1}^{j}, x_{1}, t_{j}\right)\right)^{T}, U^{j}=$ $\left(u_{M-1}^{j}, u_{M-2}^{j}, \ldots, u_{1}^{j}\right)^{T}$,

$$
\begin{aligned}
& \emptyset_{1}=\left(\varphi_{1}\left(x_{1}\right), \varphi_{1}\left(x_{2}\right), \ldots, \varphi_{1}\left(x_{N}\right)\right)^{T}, \\
& \emptyset_{2}=\left(\varphi_{2}\left(x_{1}\right), \varphi_{2}\left(x_{2}\right), \ldots, \varphi_{2}\left(x_{N}\right)\right)^{T},
\end{aligned}
$$

and $A^{j}=\left(a_{n m}^{j}\right)$ is a matrix with the following coefficients:

$$
\begin{gathered}
a_{n m}^{j}= \begin{cases}Q_{n}^{j} R_{n}^{j} G_{n-1}^{j}, & \text { where } m=1, \\
Q_{n}^{j} R_{n}^{j}\left(G_{n-m}^{j}-2 G_{n-m+1}^{j}+\theta G_{n-m+2}^{j}\right), & \text { where } m \leq n, \\
2-H_{n}^{j}+Q_{n}^{j} R_{n}^{j}\left(\theta G_{1}^{j}-2 G_{0}^{j}\right), & \text { where } m=n+1, \\
Q_{n}^{j} R_{n}^{j} G_{0}^{j}, & \text { where } m=n+2, \\
0, & \text { where } m>n+2,\end{cases} \\
\quad \theta=\left\{\begin{array}{lll}
0, & \text { where } m=2, & \\
1, & \text { otherwise, }
\end{array}\right.
\end{gathered}
$$

for $n=1,2, \ldots, K-1$, and $m=1,2, \ldots, K-1$. Also, we note that

$$
\|A\|_{\infty}=\max _{1 \leq n \leq K} \sum_{m=1}^{K}\left|a_{n m}\right|=\max _{1 \leq n \leq K}\left\{2-H_{i}^{n}\right\}=2-H_{i}^{0},
$$

then $\|A\|_{\infty}=1$.

Lemma 2. The coefficients $G_{k}^{j}$ and $H_{i}^{k}$ satisfy the following conditions:

(1) $G_{0}^{j}=1$, and $H_{i}^{0}=1$,

(2) $G_{k}^{j}>G_{k+1}^{j}$, and $H_{i}^{k}>H_{i}^{k+1}$, for $k=0,1, \ldots$

\section{The Stability Analysis and}

the Truncation Error

Let us consider $W^{j+1}$ and $U^{j+1}$ to be two different numerical solutions of (15) with initial values given by $W^{0}$ and $U^{0}$, respectively.

Theorem 3. The explicit method approximation defined by (15) to the variable order space-time wave equation (2) is unconditionally stable, that is,

$$
\left|W^{j+1}-U^{j+1}\right| \leq C\left|W^{0}-U^{0}\right|, \quad \text { for any } j .
$$

Proof. Let us define $W^{j+1}-U^{j+1}=\varepsilon^{j+1}$. From (15) we have

$$
\begin{aligned}
\varepsilon_{i}^{j+1}= & A^{j} \varepsilon_{i}^{j} \\
& -\sum_{k=2}^{M-2}\left(H_{i}^{k-2}-2 H_{i}^{k-1}+H_{i}^{k}\right) \varepsilon_{i}^{j-k+1} \\
& -\left(H_{i}^{j-2}-2 H_{i}^{j-1}\right) \varepsilon_{i}^{1}-H_{i}^{j-1} \varepsilon_{i}^{0}+F_{\varepsilon}^{j},
\end{aligned}
$$

where

$$
\begin{aligned}
F_{\varepsilon}^{j}= & \left(Q_{m-1}^{j} f\left(u_{m-1}^{j}, x_{m-1}, t_{j}\right)\right. \\
& -Q_{m-1}^{j} f\left(w_{m-1}^{j}, x_{m-1}, t_{j}\right), \ldots, Q_{1}^{j} f\left(u_{1}^{j}, x_{1}, t_{j}\right) \\
& \left.-Q_{1}^{j} f\left(w_{1}^{j}, x_{1}, t_{j}\right)\right)^{T} \\
\leq & \left(Q_{m-1}^{j} L_{m-1}^{j} \varepsilon_{m-1}^{j}, \ldots, Q_{1}^{j} L_{1}^{j} \varepsilon_{1}^{j}\right)^{T}=\Delta F^{j} \varepsilon^{j},
\end{aligned}
$$

and $\Delta F^{j}=\operatorname{diag}\left(Q_{m-1}^{j} L_{m-1}^{j}, \ldots, Q_{1}^{j} L_{1}^{j}\right)^{T}$.

Noting that $\left|L_{i}^{j}\right| \leq L$, for any $i, j$.

Let $\bar{Q}=\max \left\{Q_{m-1}^{j}, \ldots, Q_{1}^{j}\right\}$. From (20), we have $\left\|A^{j}+\Delta F^{j}\right\|_{m} \leq(2+\bar{Q} L)$, then

$$
\begin{aligned}
\left\|\varepsilon_{i}^{j+1}\right\|_{\infty} \leq & \left\|A^{j}+\Delta F^{j}\right\|_{\infty}\left\|\varepsilon_{i}^{j}\right\|_{\infty} \\
& +\sum_{k=2}^{M-2}\left(H_{i}^{k-2}-2 H_{i}^{k-1}+H_{i}^{k}\right)\left\|\varepsilon_{i}^{j-k+1}\right\|_{\infty} \\
& +\left(H_{i}^{j-2}-2 H_{i}^{j-1}\right)\left\|\varepsilon_{i}^{1}\right\|_{\infty}+H_{i}^{j-1}\left\|\varepsilon_{i}^{0}\right\|_{\infty} .
\end{aligned}
$$

Now, we analyze the stability via mathematical induction [10]. From (14) we have $\left\|\varepsilon_{i}^{1}\right\|_{\infty} \leq C\left\|\varepsilon_{i}^{0}\right\|_{\infty}$, where $C$ is a constant.

Now, assume that $\left\|\varepsilon_{i}^{j}\right\|_{\infty} \leq C\left\|\varepsilon_{i}^{0}\right\|_{\infty}$, then from (22), we have

$$
\begin{aligned}
\left\|\varepsilon_{i}^{j+1}\right\|_{\infty} \leq & C(2+\bar{Q} L)\left\|\varepsilon_{i}^{0}\right\|_{\infty} \\
& +\sum_{k=2}^{M-2}\left(H_{i}^{k-2}-2 H_{i}^{k-1}+H_{i}^{k}\right) C\left\|\varepsilon_{i}^{0}\right\|_{\infty} \\
& +C\left(H_{i}^{j-2}-2 H_{i}^{j-1}\right)\left\|\varepsilon_{i}^{0}\right\|_{\infty} \\
& +H_{i}^{j-1}\left\|\varepsilon_{i}^{0}\right\|_{\infty} \leq C_{1}\left\|\varepsilon_{i}^{0}\right\|_{\infty} .
\end{aligned}
$$

Then, the theorem holds.

Lemma 4. Let

$$
\begin{aligned}
{ }_{0} \bar{D}_{x}^{\propto\left(x_{i}, t_{j}\right)} u\left(x_{i}, t_{j}\right)= & \frac{h^{-\propto\left(x_{i}, t_{j}\right)}}{\Gamma\left(3-\propto\left(x_{i}, t_{j}\right)\right)} \\
& \times \sum_{k=0}^{j-1} G_{i}^{j}\left(u_{i-k+1}^{j}-2 u_{i-k}^{j}+u_{i-k+1}^{j}\right)
\end{aligned}
$$

be a smooth function; then

$$
\left|\bar{D}_{x}^{\propto\left(x_{i}, t_{j}\right)} u\left(x_{i}, t_{j}\right)-D_{x}^{\propto\left(x_{i}, t_{j}\right)} u\left(x_{i}, t_{j}\right)\right|=O(h) .
$$




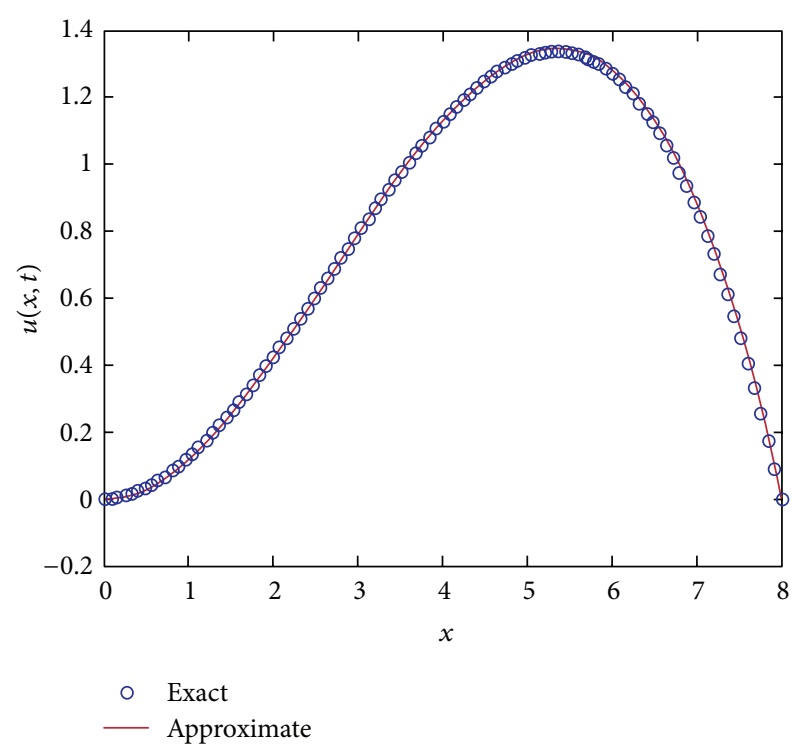

FIGURE 1

Proof. In terms of standard centered difference formula, we have

$$
\begin{aligned}
& { }_{0} \bar{D}_{x}^{\propto\left(x_{i}, t_{j}\right)} u\left(x_{i}, t_{j}\right) \\
& =\frac{h^{2-\propto\left(x_{i}, t_{j}\right)}}{\Gamma\left(3-\propto\left(x_{i}, t_{j}\right)\right)} \\
& \times \sum_{j=0}^{k-1} G_{i}^{j}\left[\frac{\partial^{2} u(x-j h, t)}{\partial z^{2}}+O\left(h^{2}\right)\right] \\
& =\frac{h^{2-\propto\left(x_{i}, t_{j}\right)}}{\Gamma\left(3-\propto\left(x_{i}, t_{j}\right)\right)} \\
& \times \sum_{j=0}^{k-1} G_{i}^{j} \frac{\partial^{2} u(x-j h, t)}{\partial z^{2}} \\
& +\frac{h^{2-\propto\left(x_{i}, t_{j}\right)} k^{2-\propto\left(x_{i}, t_{j}\right)}}{\Gamma\left(3-\propto\left(x_{i}, t_{j}\right)\right)} O\left(h^{2}\right) \\
& =\frac{h^{2-\propto\left(x_{i}, t_{j}\right)}}{\Gamma\left(3-\propto\left(x_{i}, t_{j}\right)\right)} \\
& \times \sum_{j=0}^{k-1} G_{i}^{j} \frac{\partial^{2} u(x-j h, t)}{\partial z^{2}} \\
& +\frac{x^{2-\propto\left(x_{i}, t_{j}\right)}}{\Gamma\left(3-\propto\left(x_{i}, t_{j}\right)\right)} O\left(h^{2}\right) \\
& =\frac{h^{2-\propto\left(x_{i}, t_{j}\right)}}{\Gamma\left(3-\propto\left(x_{i}, t_{j}\right)\right)} \\
& \times \sum_{j=0}^{k-1} G_{i}^{j} \frac{\partial^{2} u(x-j h, t)}{\partial z^{2}}+O\left(h^{2}\right) .
\end{aligned}
$$

By the integral mean value theorem, we have

$$
\begin{aligned}
{ }_{0} D_{x}^{\propto\left(x_{i}, t_{j}\right)} u\left(x_{i}, t_{j}\right)= & \frac{1}{\Gamma\left(2-\propto\left(x_{i}, t_{j}\right)\right)} \\
& \times \sum_{j=0}^{k-1} \int_{j h}^{(j+1) h} z^{1-\propto\left(x_{i}, t_{j}\right)} \frac{\partial^{2} u(x-z, t)}{\partial z^{2}} d z \\
= & \frac{h^{2-\propto\left(x_{i}, t_{j}\right)}}{\Gamma\left(3-\propto\left(x_{i}, t_{j}\right)\right)},
\end{aligned}
$$

where $\xi_{j} \in[j h,(j+1) h]$. Combining the pervious two formulae, we have

$$
\begin{aligned}
\left|\bar{D}_{x}^{\propto\left(x_{i}, t_{j}\right)} u\left(x_{i}, t_{j}\right)-D_{x}^{\propto\left(x_{i}, t_{j}\right)} u\left(x_{i}, t_{j}\right)\right| & \mid \frac{h^{2-\alpha\left(x_{i}, t_{j}\right)}}{\Gamma\left(3-\alpha\left(x_{i}, t_{j}\right)\right)} \\
& \times \sum_{j=0}^{k-1} G_{i}^{j}\left[\frac{\partial^{2} u(x-j h, t)}{\partial z^{2}}-\frac{\partial^{2} u\left(x-\xi_{j}, t\right)}{\partial z^{2}}\right] \\
= & \left|\frac{h^{2-\alpha\left(x_{i}, t_{j}\right)}}{\Gamma\left(3-\alpha\left(x_{i}, t_{j}\right)\right)} \sum_{j=0}^{k-1} G_{i}^{j} \cdot O(h)+O\left(h^{2}\right)\right| \\
= & \left|\frac{h^{2-\alpha\left(x_{i}, t_{j}\right)} k^{2-\alpha\left(x_{i}, t_{j}\right)}}{\Gamma\left(3-\alpha\left(x_{i}, t_{j}\right)\right)} \cdot O(h)+O\left(h^{2}\right)\right| \\
= & O(h)+O\left(h^{2}\right)=O(h) .
\end{aligned}
$$

Now, by using Lemma 4, we can derive the truncation error of explicit finite difference scheme (14). It has a local truncation error of $O(\tau)$ (from the left side) and $O(h)$ (from the right side).

Remark 5. The pervious explicit method was shown to be stable. This method is consistent with a local truncation error which is $O(\tau)+O(h)$. Therefore, according to the Lax Equivalence Theorem [2], it converges at this rate.

\section{Numerical Examples}

Example 1. Consider the following variable-order linear fractional wave equation:

$$
\begin{aligned}
\frac{\partial^{\beta(x, t)} u(x, t)}{\partial \tau^{\beta(x, t)}}= & -0.5 \cos \left(\frac{\propto(x, t) \pi}{2}\right) \\
& \times \frac{\partial^{\propto(x, t)} u(x, t)}{\partial x^{\propto(x, t)}}+f(u, x, t),
\end{aligned}
$$




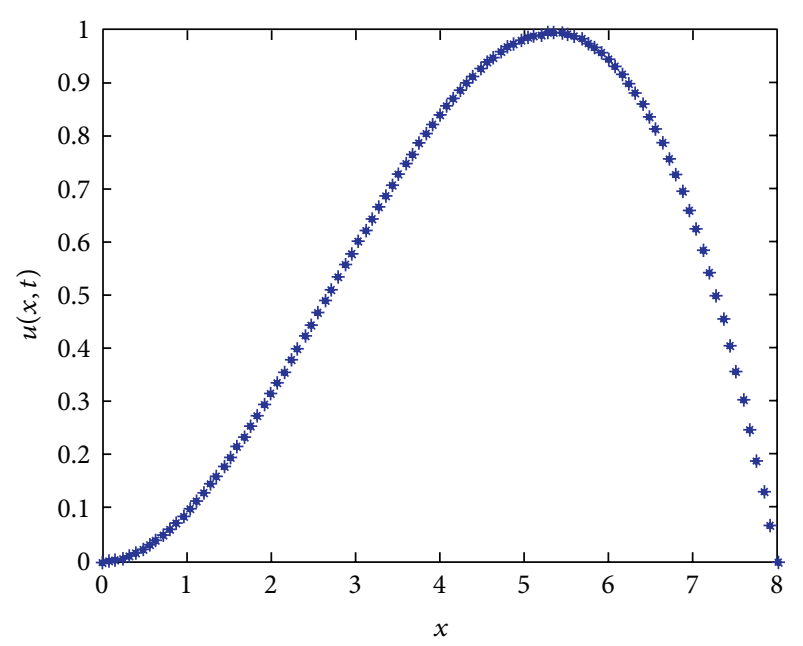

(a)

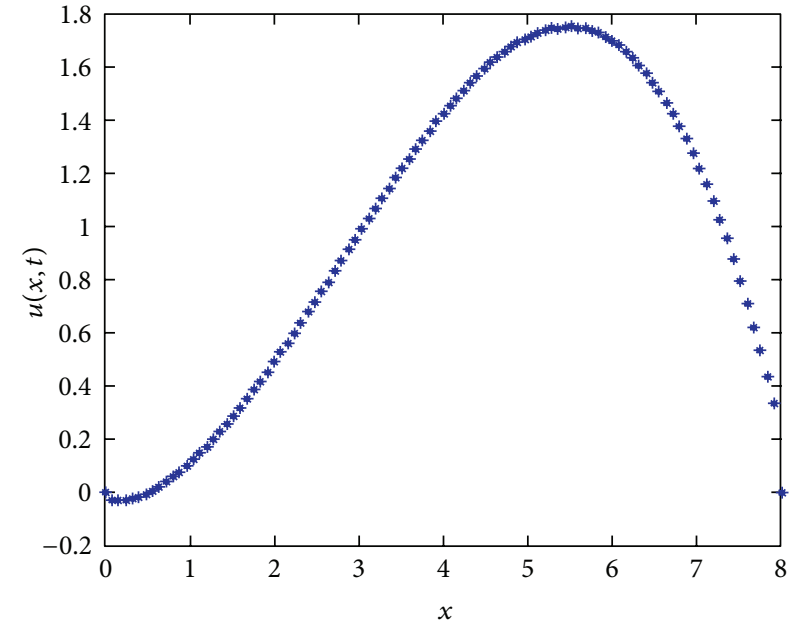

(b)

Figure 2

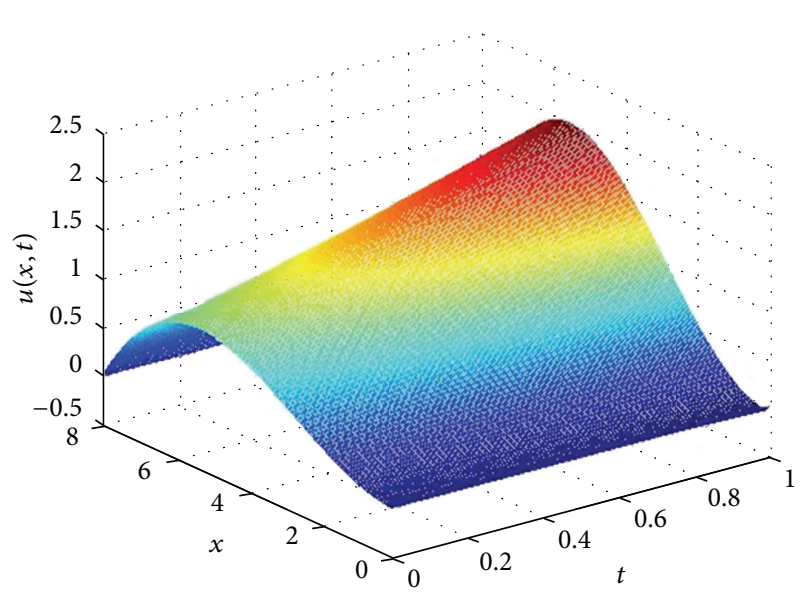

Figure 3

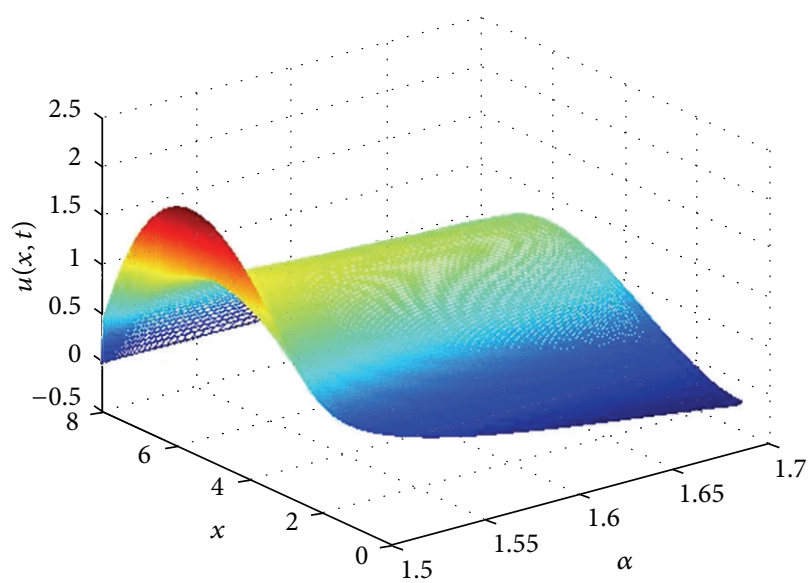

FIGURE 4

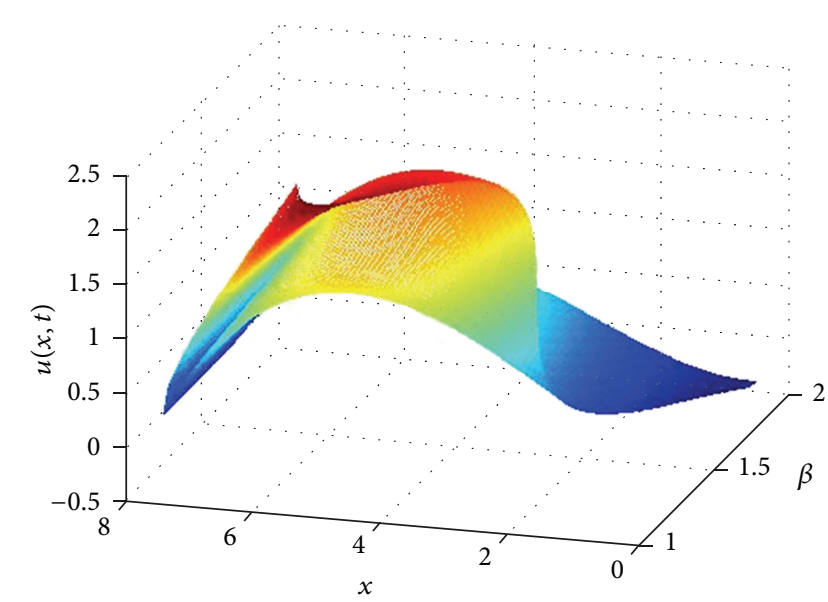

FIGURE 5

In Figures 3, 4, and 5, respectively, we report the approximate solutions in three dimensions, where the axis's are $(t, x, u),($ alfa, $x, u)$, and (beta, $x, u)$, respectively. 

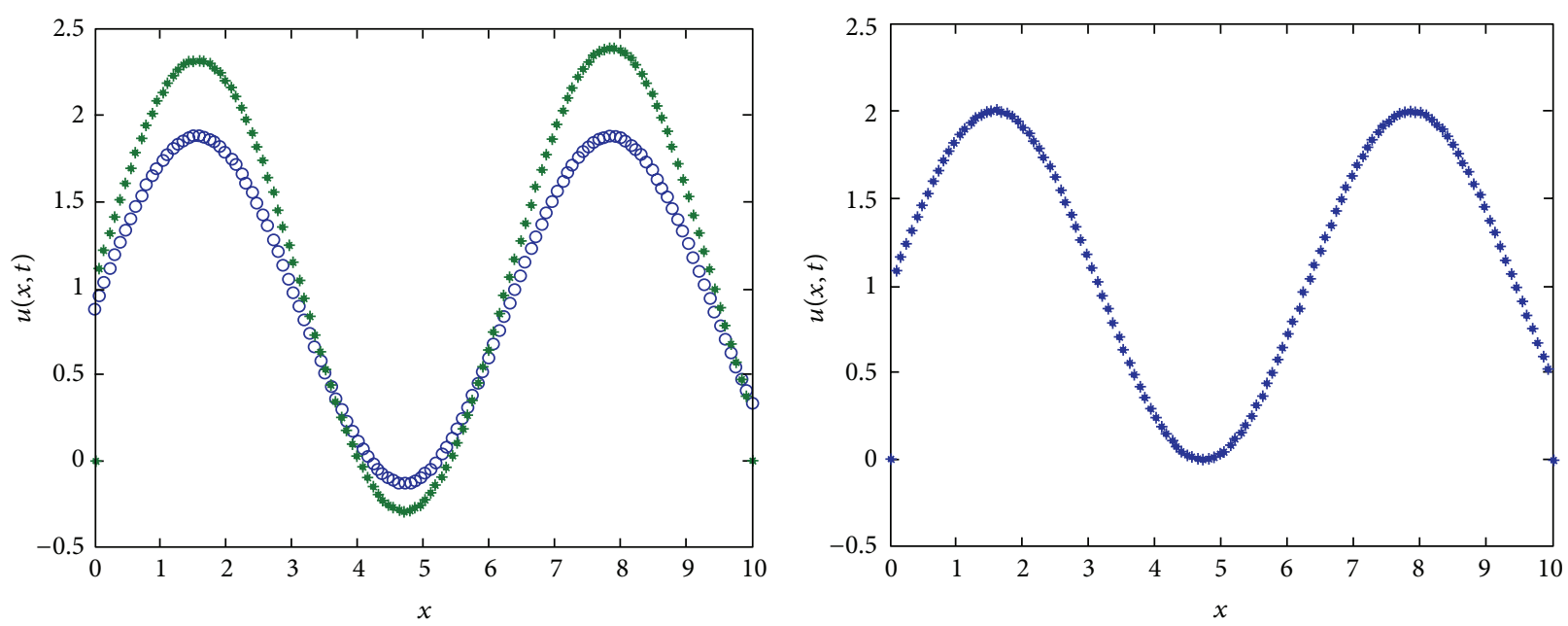

○ $\alpha=\beta=2$

* $\alpha, \beta$ are variable

(a)

(b)

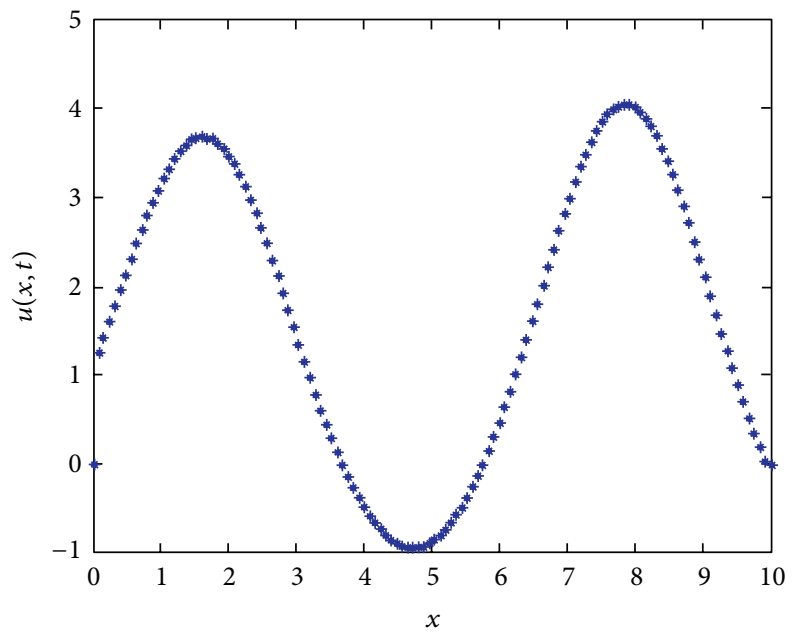

(c)

Figure 6

Example 2. Consider the following variable-order nonlinear fractional wave equation:

$$
\frac{\partial^{\beta(x, t)} u(x, t)}{\partial t^{\beta(x, t)}}=2 \cos t \frac{\partial^{\propto(x, t)} u(x, t)}{\partial x^{\propto(x, t)}}+f(u, x, t),
$$

with $\propto(x, t)=2-\cos ^{2}(x) \sin ^{2}(t), \beta(x, t)=1.8+0.5 e^{-(x t)^{2}-1}$ and $u(x, 0)=\varphi(x)=1+\sin x, u_{t}(x, 0)=\Psi(x)=0$, where $0 \leq x \leq 10, T=1$, and $f(u, x, t)=u^{2}-\sin ^{2}(x)-\cos ^{2}(t)$. $\propto=2$

This problem has the following exact solution, when

$$
u(x, t)=\sin x+\cos t
$$

In Figure 6(a), we report the numerical solution when $\propto$ $(x, t), \beta(x, t)$ are variables at $t=0.52$ and the exact solutions when $\propto=\beta=2$.

In Figures 6(b) and 6(c), we report the approximate solution at $t=0.052$ and $t=0.78$, respectively.

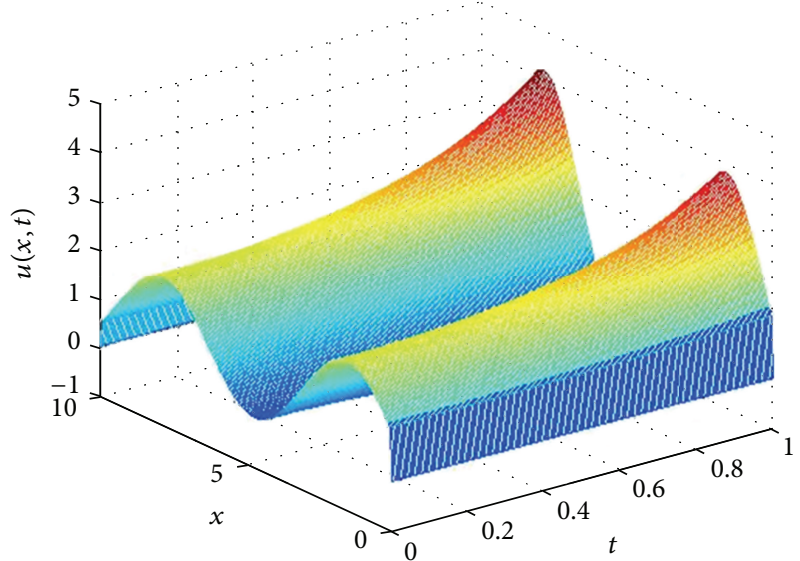

Figure 7

Figures 7, 8, and 9 show the approximate solution in three dimensions, where the axes are $(t, x, u),($ alfa, $x, u)$ and (beta, $x, u$ ), respectively. 


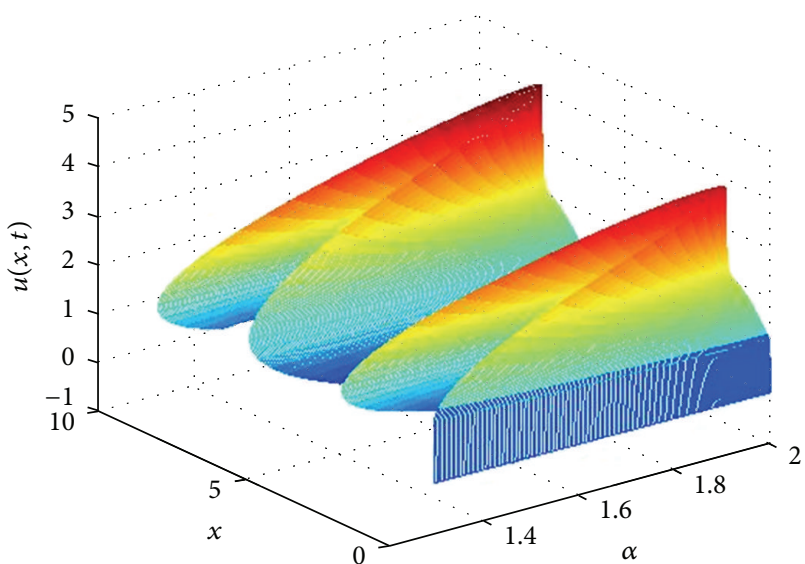

FIGURE 8

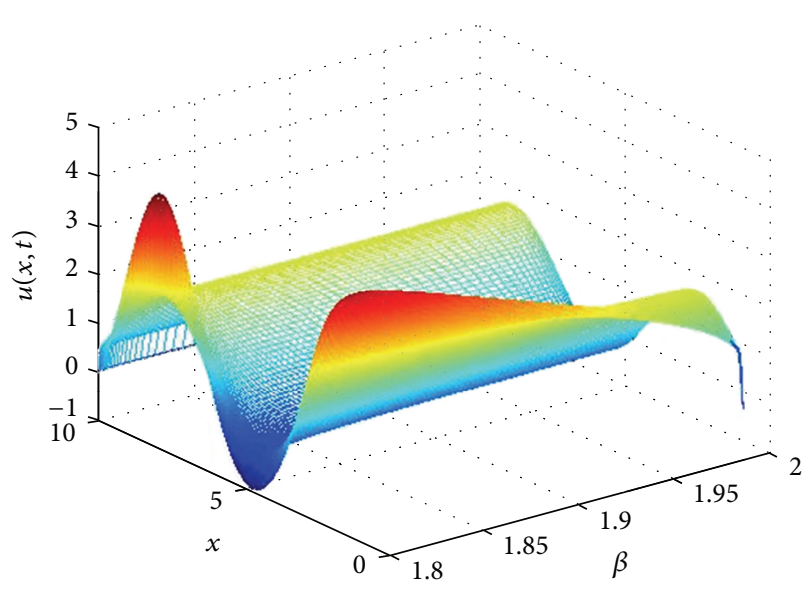

Figure 9

\section{Conclusions}

In this paper, numerical studies using a simple explicit FDM for solving the variable order space-time wave equation are presented. The stability analysis and the truncation error of the proposed method are proved. Some test examples are given, and the results obtained by the method are compared with the exact solutions in integer order cases. Several figures are presented to simulate the solutions behaviors when the variable orders change with respect to space and time. The comparison certifies that FDM gives good results. Summarizing these results, we can say that the finite difference method in its general form gives reasonable calculations, easy to use, and can be applied for the variable order differential equations in general form. All results were obtained by using MATLAB version 7.6.0 (R2008a).

\section{References}

[1] I. Podlubny, Fractional Differential Equations, Academic Press, 1999.

[2] R. D. Richtmyer and K. W. Morton, Difference Methods for Initial-Vlue Problems, Krieger, Malabar, Fla, USA, 2nd edition, 1994.
[3] R. Lin, F. Liu, V. Anh, and I. Turner, "Stability and convergence of a new explicit finite-difference approximation for the variable-order nonlinear fractional diffusion equation," Applied Mathematics and Computation, vol. 212, no. 2, pp. 435-445, 2009.

[4] C. F. Lorenzo and T. T. Hartley, "A Solution to the fundamental linear fractional order differential equation," NASA/TP 1998208693, 1998.

[5] C. F. Lorenzo and T. T. Hartley, "The vector linear fractional initialization problem," NASA/TP 1999-208919, 1999.

[6] N. H. Sweilam, M. M. Khader, and R. F. Al-Bar, "Numerical studies for a multi-order fractional differential equation," Physics Letters A, vol. 371, no. 1-2, pp. 26-33, 2007.

[7] N. H. Sweilam and M. M. Khader, "A Chebyshev pseudospectral method for solving fractional-order integrodifferential equations," The ANZIAM Journal, vol. 51, no. 4, pp. 464-475, 2010.

[8] N. H. Sweilam, M. M. Khader, and A. M. Nagy, "Numerical solution of two-sided space-fractional wave equation using finite difference method," Journal of Computational and Applied Mathematics, vol. 235, no. 8, pp. 2832-2841, 2011.

[9] N. H. Sweilam, M. M. Khader, and H. M. Almarwm, "Numerical studies for the variable-order nonlinear fractional wave equation," Fractional Calculus and Applied Analysis, vol. 15, no. 4, pp. 669-683, 2012.

[10] S. Shen and F. Liu, "Error analysis of an explicit finite difference approximation for the space fractional diffusion equation with insulated ends," The ANZIAM Journal, vol. 46, pp. C871-C887, 2005.

[11] S. G. Samko and B. Ross, "Integration and differentiation to a variable fractional order," Integral Transforms and Special Functions, vol. 1, no. 4, pp. 277-300, 1993.

[12] D. Ingman, J. Suzdalnitsky, and M. Zeifman, "Constitutive dynamic-order model for nonlinear contact phenomena," Journal of Applied Mechanics, Transactions ASME, vol. 67, no. 2, pp. 383-390, 2000.

[13] D. Ingman and J. Suzdalnitsky, "Control of damping oscillations by fractional differential operator with time-dependent order," Computer Methods in Applied Mechanics and Engineering, vol. 193, no. 52, pp. 5585-5595, 2004.

[14] F. Liu, P. Zhuang, V. Anh, and I. Turner, "A fractional-order implicit difference approximation for the space-time fractional diffusion equation," The ANZIAM Journal, vol. 47, pp. C48-C68, 2006.

[15] C. F. Lorenzo and T. T. Hartley, "Initialized fractional calculus," International Journal of Applied Mathematics, vol. 3, no. 3, pp. 249-265, 2000.

[16] C. F. Lorenzo and T. T. Hartley, "Variable order and distributed order fractional operators," Nonlinear Dynamics, vol. 29, no. 14, pp. 57-98, 2002.

[17] C. F. M. Coimbra, "Mechanics with variable-order differential operators," Annalen der Physik, vol. 12, no. 11-12, pp. 692-703, 2003.

[18] C. F. M. Coimbra and L. E. S. Ramirez, "A variable order constitutive relation for viscoelasticity," Annalen der Physik, vol. 16, no. 7-8, pp. 543-552, 2007.

[19] C. F. M. Coimbra, C. M. Soon, and M. H. Kobayashi, “The variable viscoelasticity oscillator," Annalen der Physik, vol. 14, no. 6, pp. 378-389, 2005. 


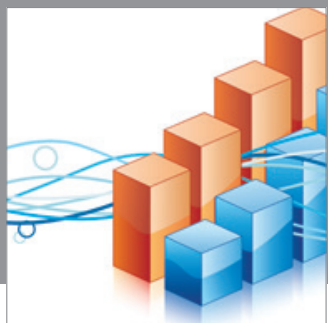

Advances in

Operations Research

mansans

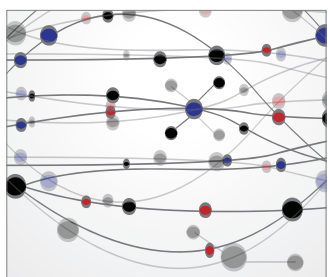

The Scientific World Journal
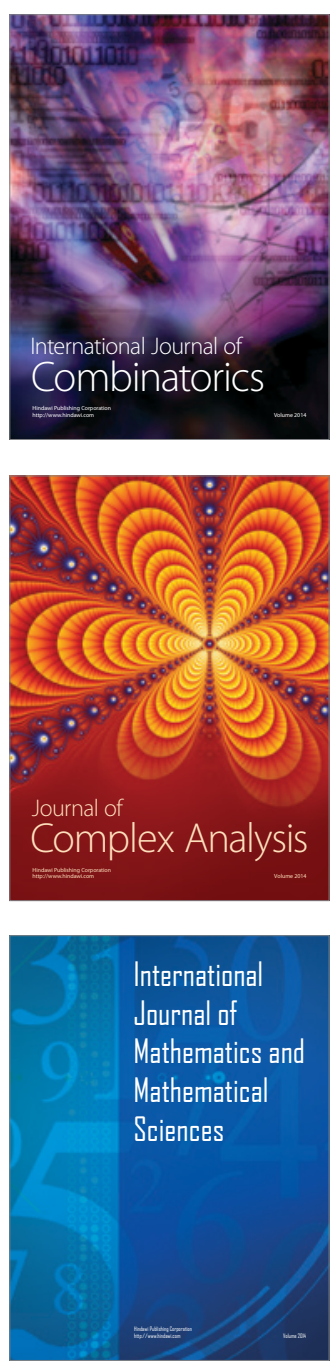
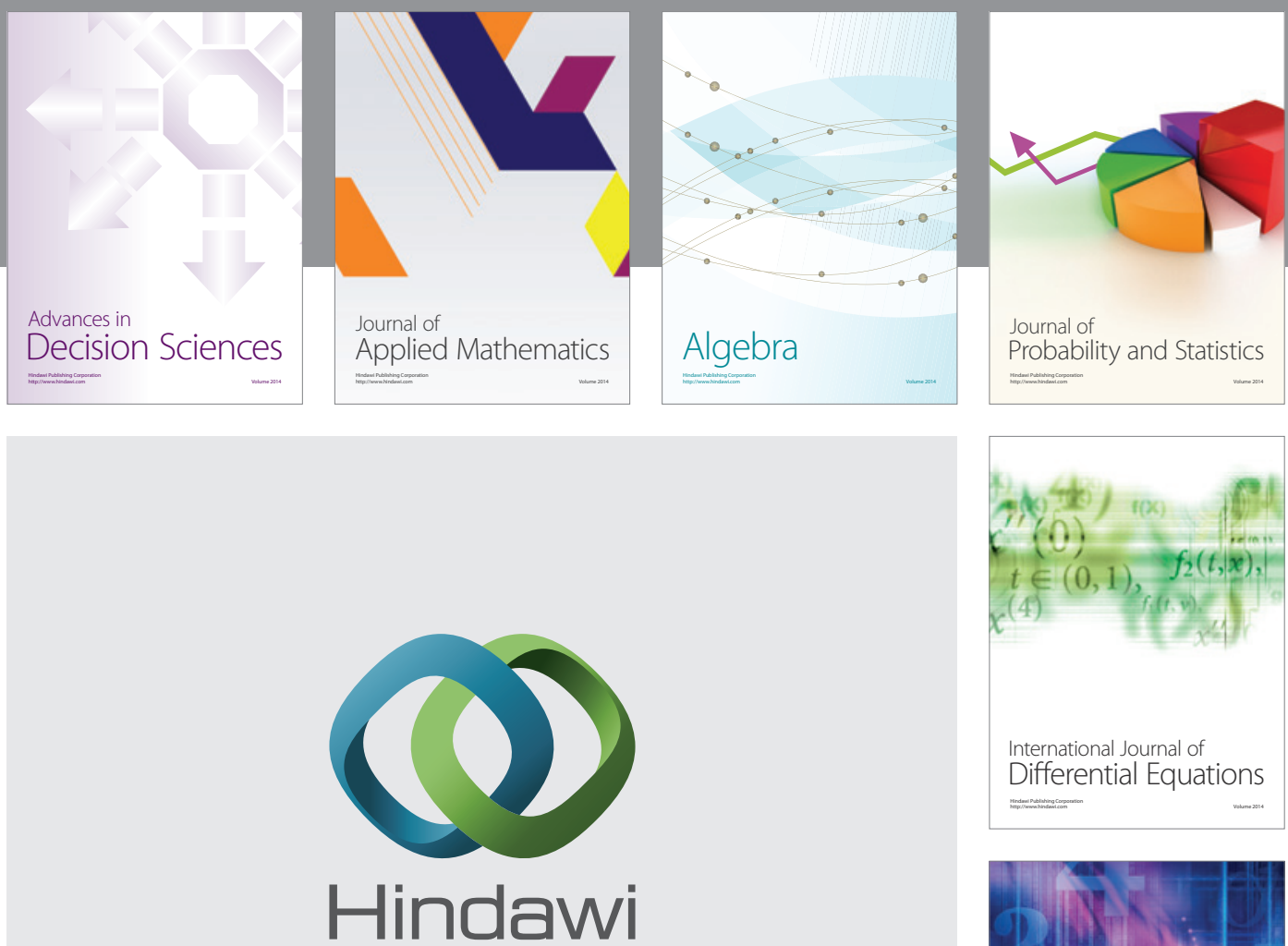

Submit your manuscripts at http://www.hindawi.com
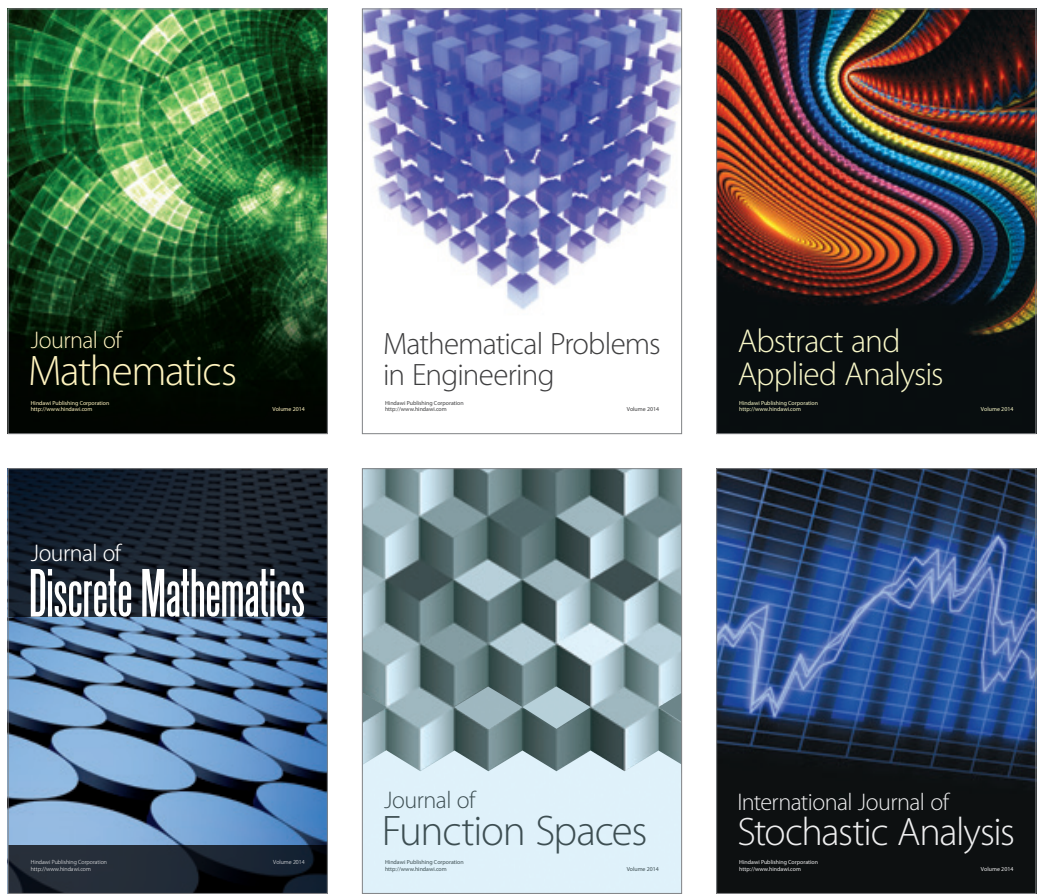

Journal of

Function Spaces

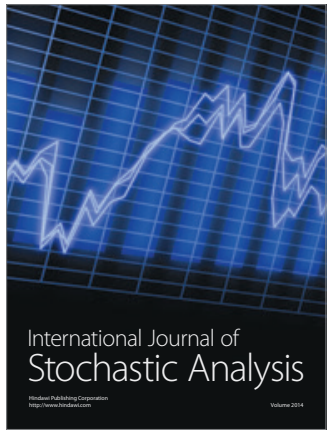

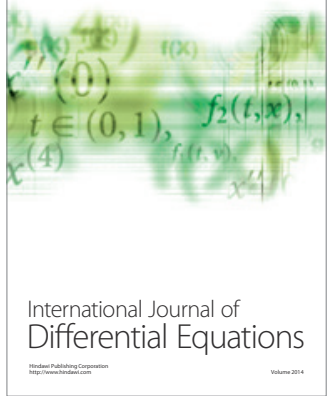
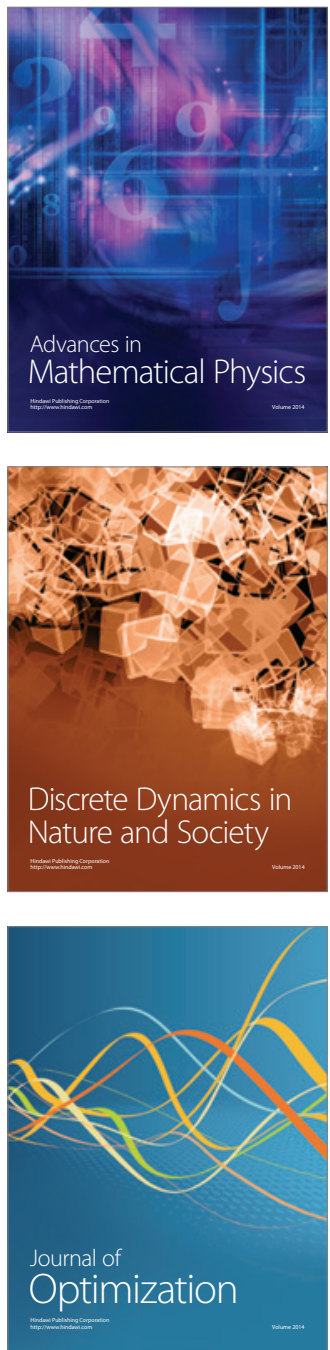